\title{
Electron Microscopic Studies of Crystallite Structures of the Collagen Model Polypeptide, (Pro-Hyp-Gly) ${ }_{10}$
}

\author{
Haruaki Eto, Kenji Okuyama, and Motowo TAKayanagi \\ Department of Applied Chemistry, Kyushu University, \\ Fukuoka 812, Japan.
}

(Received May 16, 1978)

\author{
KEY WORDS (Pro-Hyp-Gly) ${ }_{10}$ / Crystallite / Electron Microscope / \\ Collagen /
}

The collagen fibril is formed from a basic unit called tropocollagen, which consists of three coiledcoil polypeptide chains. It is known that several types of collagen fibrils can be reconstructed from the acidic solution of tropocollagen under different conditions. The addition of appropriate salt to the solution forms the native-type fibrils, in which neighboring tropocollagens are staggered by $1 \mathrm{D}$ $(\mathrm{D} \simeq 67 \mathrm{~nm})$. The addition of glycoprotein induces formation of the "fibrous-long-spacing" structure, whose period is about $280 \mathrm{~nm}$. In this fibril, tropocollagens align in anti-parallel and in register. The addition of adenosine triphosphoric acid leads to the formation of the "segment-longspacing" structure, which has a length of about $280 \mathrm{~nm}$. In this fibril, the arrangement of tropocollagens is parallel and in register.

In this paper, we present the structure of a synthetic polypeptide (Pro-Hyp-Gly) ${ }_{10}$ whose molecular weight is monodispersed. (Pro-Hyp-Gly) ${ }_{10}$ was obtained from the Protein Research Foundation, Osaka. The transition temperature of (ProHyp-Gly $)_{10}$ in aqueous acetic acid is about $331 \mathrm{~K}$; below this temperature, (Pro-Hyp-Gly) ${ }_{10}$ forms a triple helix similar to collagen. ${ }^{1}$ The experimental method was as follows. Polypeptides were dissolved in $0.05-\mathrm{mol} / l$ acetic acid at $276 \mathrm{~K}$ at the concentration of $1 \mathrm{mg} / \mathrm{m} l$. Negative staining was carried out by the mixed dropping method with $2-\%$ phosphotungstic acid $(\mathrm{pH}=7.0)$ : a few drops of the mixed solution allowed to fall on the mesh coated with the carbon film and the excess fluid is blotted off with filter paper. Positive staining of carboxyl-terminal groups was performed by the mixed dropping method with 1-\% uranyl acetate: a few drops of the mixture are applied to the mesh and the excess fluid is blotted off with filter paper; the mesh is then rinsed with distilled water. The mesh was examined in an Hitachi $\mathrm{H}-500$ electron microscope at a magnification of 50,000 or 100,000 .

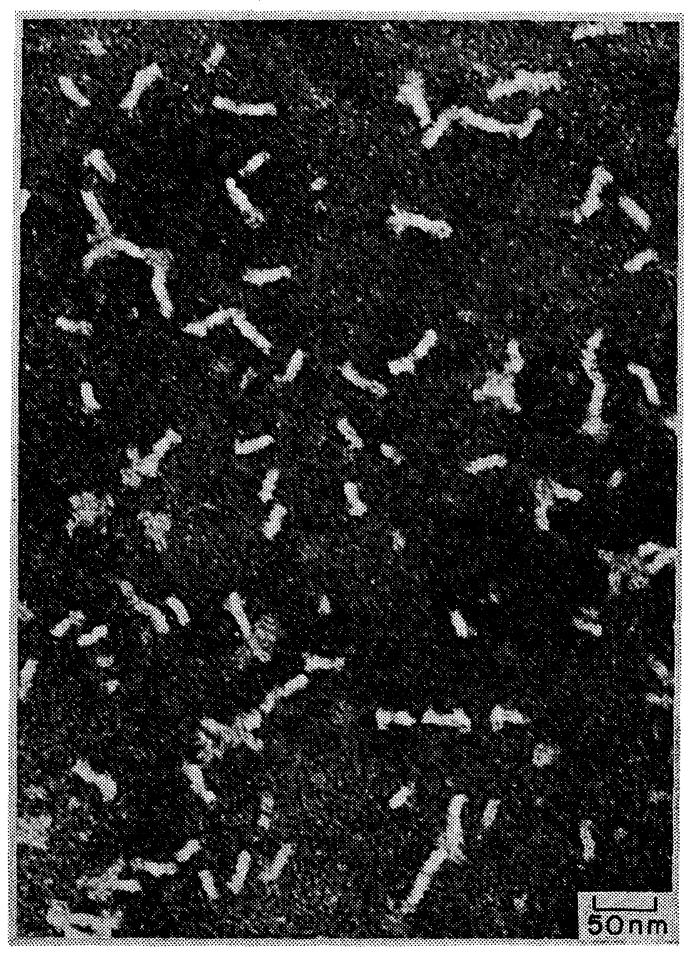

Figure 1. Electron micrograph of the crystallites of (Pro-Hyp-Gly) ${ }_{10}$ negatively stained with phosphotungstic acid. 
The accelerating voltage was $75 \mathrm{kV}$.

Figure 1 shows an electron micrograph of the crystallites of polypeptides (Pro-Hyp-Gly) ${ }_{10}$ negatively stained with phosphotungstic acid. The crystallites show comparatively regular dimensions of $8.90 \pm 0.14 \mathrm{~nm}$. Figure 2 shows an electron micrograph of the crystallites positively stained with uranyl acetate, in which the carboxyl-terminal groups positively stained by uranyl acetate form two lines with the distance of $10.23 \pm 0.25 \mathrm{~nm}$. These regular dimensions of the crystallites observed by both negative and positive staining methods are close to the molecular dimension of (Pro-Hyp-Gly $)_{10}, \quad 8.6 \mathrm{~nm} \quad(=0.29 \mathrm{~nm} /$ residue $\times 30$

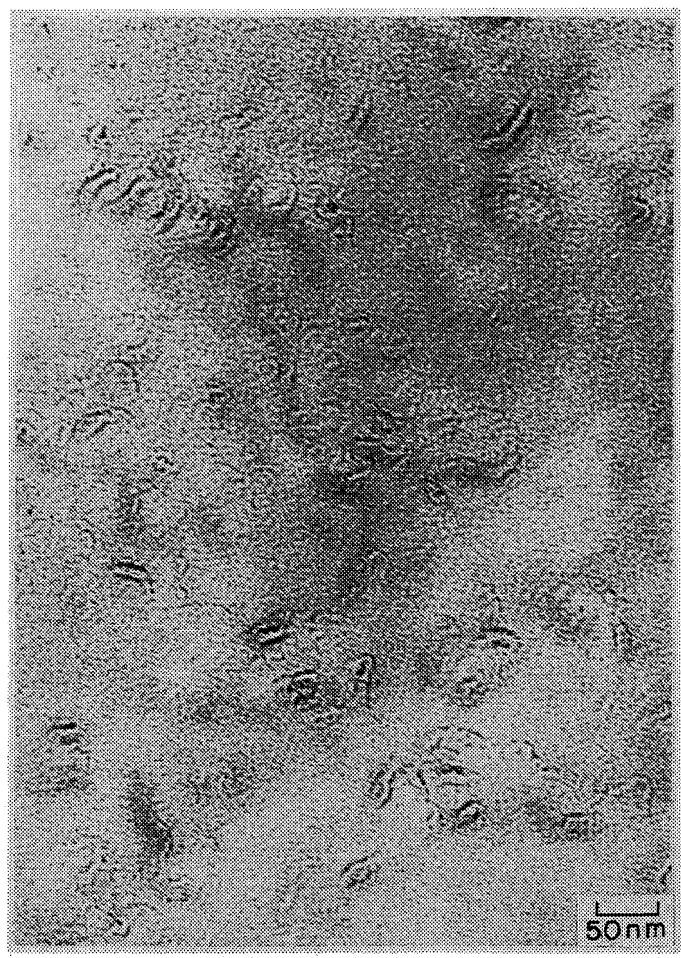

Figure 2. Electron micrograph of the crystallites of (Pro-Hyp-Gly) ${ }_{10}$ positively stained with uranyl acetate.

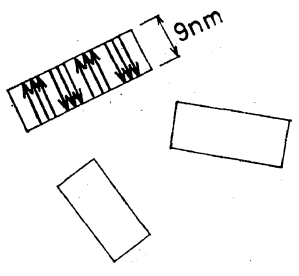

Negative staining

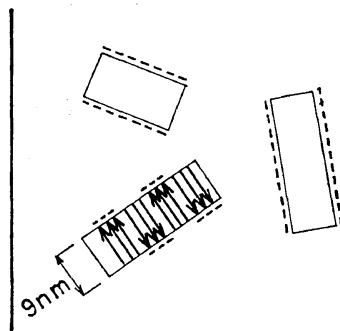

Positive staining
Figure 3. Schematic diagrams of the arrangement of triple-helical chains of (Pro-Hyp-Gly) $)_{10}$ in the crystallites.

residues), which was expected from the result of the X-ray structure analysis of (Pro-Pro-Gly) ${ }_{10} .^{2}$ Therefore, it was concluded that the crystallites are formed by the side-by-side association of triple helices (Figure 3). Since the polypeptide chain represented by an arrow in Figure 3 is expected to be packed in a parallel fashion in a triple helix by analogy with the triple helix of (Pro-Pro-Gly) ${ }_{10}$, the results of positive staining made in register as seen in Figure 3 show that (Pro-Hyp-Gly) ${ }_{10}$ triple helices are packed in the crystallites in an antiparallel fashion. The aggregate state of polypeptides in this case is similar to the "fibrous-longspacing" structure of collagen fibril. These results agree with those for the polypeptide (ProPro-Gly) $)_{10} .^{3}$

\section{REFERENCES}

1. S. Sakakibara, K. Inouye, K. Shudo, Y. Kishida, Y. Kobayashi, and D. J. Prockop, Biochem. Biophys. Acta, 303, 198 (1973).

2. K. Okuyama, N. Tanaka, T. Ashida, M. Kakudo, S. Sakakibara, and Y. Kishida, J. Mol. Biol., 72, 571 (1972).

3. B. R. Olsen, R. A. Berg, S. Sakakibara, Y. Kishida, and D. J. Prockop, J. Mol. Biol., 57, 589 (1971). 